\title{
Experimental observation of slow mode dispersion in photonic crystal coupled-cavity waveguides
}

\author{
Jana Jágerská, ${ }^{1, *}$ Nicolas Le Thomas, ${ }^{1}$ Vasily Zabelin, ${ }^{1}$ Romuald Houdré, ${ }^{1}$ \\ Wim Bogaerts, ${ }^{2}$ Pieter Dumon, ${ }^{2}$ and Roel Baets ${ }^{2}$ \\ ${ }^{1}$ Institut de Photonique et dElectronique Quantiques, Ecole Polytechnique Fédérale de Lausanne (EPFL), \\ Station 3, CH-1015 Lausanne, Switzerland \\ ${ }^{2}$ Department of Information Technology, Photonics Research Group, Ghent University-Interuniversity \\ Microelectronics Center, Ghent, Belgium \\ *Corresponding author: jana.jagerska@epfl.ch
}

Received October 14, 2008; revised December 10, 2008; accepted December 11, 2008; posted December 22, 2008 (Doc. ID 102719); published January 29, 2009

\begin{abstract}
We experimentally investigate the dispersion curve of an integrated silicon-on-insulator coupled-cavity waveguide in a photonic crystal environment using a technique based on far-field imaging. We show that a chain of eight coupled cavities of a moderate $Q$ factor can form a continuous dispersion band characterized by extremely flat dispersion and a group index of $105 \pm 20$ within a $2.6 \mathrm{~nm}$ wavelength range. The experimental results are well reproduced by theoretical calculations based on the guided-mode expansion method. (C) 2009 Optical Society of America

OCIS codes: $230.4555,230.5298$
\end{abstract}

Evanescently coupled optical resonators concatenated in a chain, so-called coupled-cavity waveguides (CCWs) [1,2], are currently regarded as a possible solution for integrated delay lines in on-chip optical circuits $[3,4]$. Their ability to slow down the light pulses relies on the storage time associated with each of the cavities that form the CCW. The periodic in-line arrangement of the cavities leads to a dispersion relation that governs the propagation properties of the guided light. A decrease of the coupling between the cavities results in a decrease of the slope of the dispersion curve, i.e., a drop in the group velocity of the guided light.

The length of the CCW has a strong impact on the dispersion properties. For a CCW of a finite length, the dispersion curve is sampled with a number of points equal to the number of the cavities $N$. If the linewidth $\delta \omega$ of the cavity mode is narrow compared to the entire bandwidth $\Delta \omega$ of the CCW divided by $N$, the optical transmission is composed of wellseparated sharp peaks $[5,6]$. In such a regime, an input pulse of bandwidth $\Delta \omega$ is transformed into its convolution with a frequency comb formed by the $\mathrm{CCW}$, and a propagating pulse is slowed down at the expense of the distortion of its shape. In the other case, i.e., if $\delta \omega \geqslant \Delta \omega / N$, the dispersion curve of the CCW is expected to be smooth and continuous and a transmission band is formed. Creation of such a band is essential for practical applications, as the optical signal can propagate without distortion only if all its spectral components are equally transmitted. Taking into account that the propagation loss of the CCW mode is scaled with $\delta \omega$, the optimum case for the management of propagation of light pulses is achieved when $\delta \omega \approx \Delta \omega / N$.

To predict the behavior of light pulses in real CCWs as well as the limitations of these structures, a precise experimental knowledge of the entire dispersion curve is crucial. However, when an entire band is transmitted instead of separated sharp peaks, the dispersion curve cannot be inferred from a simple transmission experiment as in [5].

In this Letter, we accurately determine the experimental dispersion curve of an integrated silicon on insulator (SOI) $\mathrm{CCW}$ in the regime of a continuous transmission. The measurement is based on a Fourier space imaging technique that analyzes the intrinsic out-of-plane losses of the CCW structure. We show that a chain of eight cavities can support a slow-light mode with group index of $105 \pm 20$ over a bandwidth as large as $0.33 \mathrm{THz}(2.6 \mathrm{~nm})$.

The coupled-cavity structure under consideration is shown in Fig. 1(a). It is based on a standard photonic crystal single-line-defect waveguide (W1), the lattice constant of which is periodically decreased in the propagation direction by $10 \mathrm{~nm}$ to create a double heterostructure. It is well established that such a structure supports resonant modes [7]. The optical cavities are formed by four successive periods of lattice constant $a=430 \mathrm{~nm}$, separated by potential barriers consisting of two periods of $a_{b}=420 \mathrm{~nm}$ [highlighted in Fig. 1(a)]. The total length of the CCW is $25.8 \mu \mathrm{m}$, and it comprises eight cavities, which are

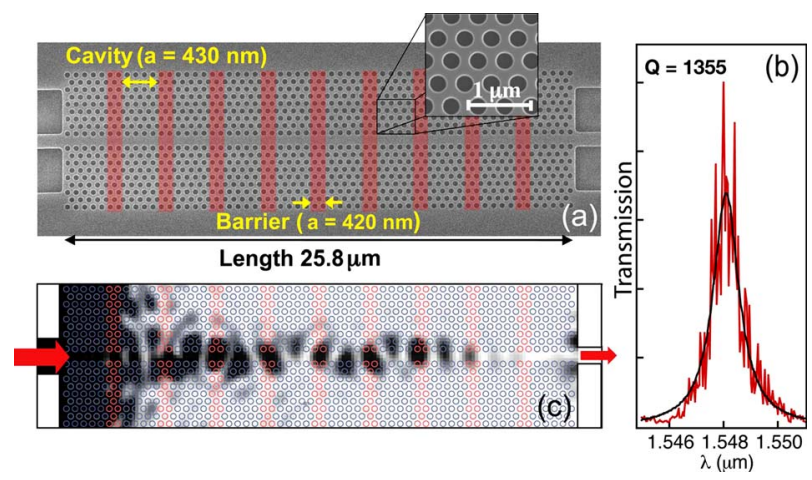

Fig. 1. (Color online) (a) Top-view SEM image of the heterostructure CCW. (b) Transmission spectrum of a single cavity. (c) Near-field intensity profile of the CCW mode recorded at $\lambda=1546.8 \mathrm{~nm}$. 
coupled to the access waveguides by an intermediate photonic crystal waveguide region of $a=430 \mathrm{~nm}$. The hole diameter of $d=286 \mathrm{~nm}$ is constant in the whole photonic crystal, corresponding to the filling factor of $f=40 \%$ in the cavities. In the present study, an experimental quality factor in the range of 1355 was measured for the cavities processed in $220 \mathrm{~nm}$ thick SOI [see Fig. 1(b)].

A dispersion diagram of the structure calculated by the guided-mode expansion (GME) method [8] is presented in Fig. 2(a) (dotted curve). At reduced frequencies larger than 0.279 , the mode dispersion is similar to the dispersion of a standard W1 waveguide, folded by the periodicity of the supercell defined by $\Lambda=4 a+2 a_{b}$. In this frequency range, the barrier regions still support a propagating mode, and the upper band represents a dispersion curve of a weakly corrugated W1 waveguide. The coupled-cavity mode appears approximately $4 \mathrm{~nm}$ below the cutoff of the "W1 mode" and is characterized by cosine-shaped dispersion and a bandwidth spanning $2.6 \mathrm{~nm}$ $(0.33 \mathrm{THz})$. Formation of such a CCW mode can be equally understood in terms of a perturbed W1 waveguide; it is a result of the anticrossing of the folded W1 modes, which are coupled owing to the periodic perturbation introduced by the barriers. This is illustrated in Fig. 2(b), which shows how the W1 mode splits and the band diagram is formed once the coupling interaction is introduced.

To experimentally probe the CCW dispersion, the sample was fabricated on an SOI material system ( $220 \mathrm{~nm}$ thick Si layer on $2 \mu \mathrm{m}$ buried oxide) using a complementary metal-oxide semiconductor compatible deep-UV lithography [9]. A 330-nm-thick resist layer with a bottom antireflective coating was patterned by $193 \mathrm{~nm}$ illumination. The resist served directly as an etch mask for inductively coupled plasma-reactive ion etching (ICP-RIE), which followed the writing process to transfer the pattern into the silicon device layer. The top view scanning elec-

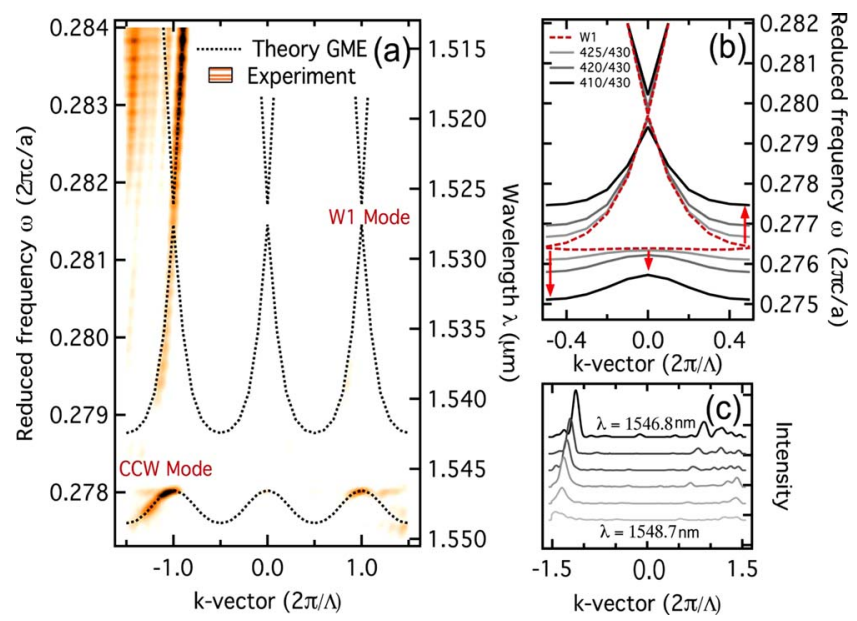

Fig. 2. (Color online) (a) Experimental dispersion diagram compared to the GME calculation. (b) Simulation of the CCW mode formation upon increasing the lattice constant difference between the cavities $(a=430 \mathrm{~nm})$ and the barriers $\left(a_{b}=430,425,420\right.$, and $\left.410 \mathrm{~nm}\right)$. (c) Line profiles of the CCW mode dispersion. tron microscopy (SEM) image of the sample surface shown in Fig. 1(a) confirms the high quality of the structure pattern. The fabricated structure was excited using a highly monochromatic cw laser of a linewidth of $\Delta \lambda<1 \mathrm{pm}$, tunable within the wavelength range of $1470-1660 \mathrm{~nm}$. TE polarized light was coupled into the sample by use of lensed fiber and adiabatically tapered access waveguides.

Unlike typical low-loss optical waveguides that support fully guided, nonradiative modes, the CCWs are intrinsically lossy. The imposed periodicity of the coupled cavity chain acts as a diffraction grating, which perturbs the guided field and scatters a part of the propagating light into the radiative continuum [10]. Although detrimental for energy performance, this permits us to study the light propagation within the structure by means of standard optical imaging. Light radiated from the sample surface was collected using a high numerical aperture objective $(\mathrm{NA}=0.9)$ and projected onto a sensitive infrared (IR) camera.

The real-space image showing the near-field intensity distribution of the CCW mode is presented in Fig. 1(c). Although the field decay along the propagation direction is significant $(\sim 3 \mathrm{~dB}$ per $10 \mu \mathrm{m})$, it can be clearly seen that all eight cavities are excited and that the light is well localized within the core of the CCW structure. The weak but apparent transversal oscillation of the near-field pattern is attributed to subnanometer structural disorder, which notably disturbs the signal propagation in the sensitive slowlight regime.

To probe the spectral properties of the $\mathrm{CCW}$ mode including the dispersion relation, we use a Fourierspace imaging technique, which was discussed in detail in $[10,11]$. By projecting the back focal plane of the collecting lens on the IR camera, we access the far-field or $k$-space image, which represents the optical Fourier transform of the near-field pattern. In such an image, every point $\left(k_{\|}, k_{\perp}\right)$ is uniquely related to a wave vector of the radiated field and, according to the wave-vector conservation, also to a wave vector of mode propagating in the structure. Hence, by recording the $k$-vector distribution for each excitation frequency $\omega$, we can retrieve the dispersion relation $\omega(k)$ of the guided field.

The experimental dispersion diagram showing both the CCW and the corrugated W1 waveguide mode is plotted in Fig. 2(a) together with the result of the GME simulation. The direct comparison demonstrates a good agreement between the theory and the measurement in respect to both the position and the slope of the investigated modes. The NA of our imaging system allows us to probe the first three Brillouin zones nearest to the center of the dispersion plane. Hence, in the dispersion map we observe three dispersion curves that are identical in shape but offset along the $k$-vector coordinate by the reciprocal vector of the coupled-cavity chain $2 \pi / \Lambda=2.45 \mu \mathrm{m}^{-1}$. Their peak intensities reflect the energy distribution in individual Brillouin zones, i.e., the relative intensities of the spectral components of the Bloch mode.

Intensity profiles of the far-field spectra of the CCW mode at different excitation wavelengths be- 
tween 1546.8 and $1548.7 \mathrm{~nm}$ are presented in Fig. 2(c). Upon increasing the frequency of excitation, the peaks follow the dispersion of the CCW mode. The linewidth of the far-field spectra does not change notably within the CCW mode bandwidth and is primarily determined by the finite length of the measured structure. The intensity of the collected light is maximum at the top of the CCW dispersion band, which is attributed to higher out-of-plane radiation loss of the symmetric CCW mode. A closer zoom on the CCW mode dispersion is given in Fig. 3, which presents the experimental dispersion curve in the second Brillouin zone normalized in intensity, together with the theoretical band diagram calculated by the GME method. Markers that depict the maxima of the experimental data help to visualize the measured dispersion curve. The experimental dispersion $\omega(k)$ is smooth enough to be numerically differentiated to obtain the group index and its frequency dependence, as shown in Fig. 3 (right). The experimental dispersion relation agrees well with the theoretical calculations until a maximum group index of approximately $n_{g}=330$. This is one of the largest experimental group index values reported for an optical waveguide, although the error bars are delicate to estimate in such a narrow energy and wave-vector range. Above this value, the CCW mode deflects from the ideal cosine-shaped dispersion and gets smoothly coupled into a fast-decaying evanescent mode that emerges from the top and the bottom of the dispersion band. The maximum achievable value of the group index $\left(n_{g}=330\right)$ is strongly limited by the structural disorder and intrinsic out-of-plane losses, which significantly modify the ideal dispersion diagram as was discussed in [12].

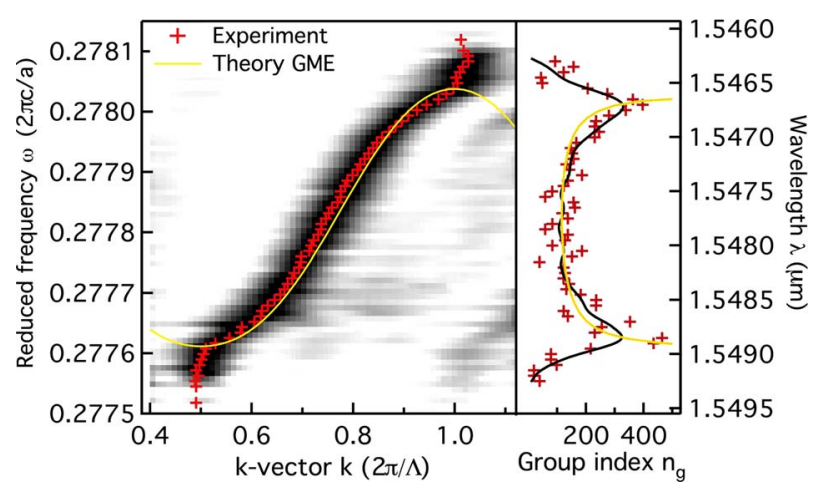

Fig. 3. (Color online) Normalized dispersion of the CCW mode (left) and its group index $n_{g}$ (right). The experimental group index was obtained by both numerical differentiation of the raw data (crosses) and data interpolated by a spline function (black curve).
To evaluate the buffering capacity of the measured device we calculate the normalized delay-bandwidth product $\left(\mathrm{DBP}_{N}\right)$ of the CCW [4], which can be expressed as a product of the group index and the bandwidth of the mode of interest. The group index does not drop below $105 \pm 20$ in the entire bandwidth of the CCW mode $(0.33 \mathrm{THz})$, which corresponds to a $\mathrm{DBP}_{N}$ as large as 0.18 . It should be remarked that within $2 \mathrm{~nm}$ of this bandwidth the dispersion of the mode is very close to linear, so the propagating pulse (of time duration down to $2 \mathrm{ps}$ ) will not be distorted owing to group-velocity dispersion.

In summary, we have presented a dispersion curve of a double heterostructure CCW probed by a technique of far-field imaging. The experimental data taken with high spectral resolution allowed us to retrieve the dispersion curve of the CCW mode with a high accuracy and calculate its group index, which was found to be larger than $105 \pm 20$ within a $2.6 \mathrm{~nm}$ wavelength range.

The authors acknowledge support from the European projects ePIXnet (IST-004525), the COST P11 and MP0702 actions, and the Swiss National Centre of Competence in Research-Quantum Photonics.

\section{References}

1. N. Stefanou and A. Modinos, Phys. Rev. B 57, 12127 (1998).

2. A. Yariv, Y. Xu, R. K. Lee, and A. Scherer, Opt. Lett. 24, 711 (1999).

3. F. Xia, L. Sekaric, and Y. Vlasov, Nat. Photonics 1, 65 (2007).

4. T. Baba, Nat. Photonics 2, 465 (2008).

5. E. Kuramochi, T. Tanabe, H. Taniyama, M. Kato, and M. Notomi, in Proceedings of the Conference on Lasers and Electro-Optics and Quantum Electronics and Laser Science Conference (CLEO/QELS'07) (2007), paper QMG2.

6. B. M. Möller, U. Woggon, and M. V. Artemyev, Opt. Express 15, 17362 (2007).

7. B.-S. Song, S. Noda, T. Asano, and Y. Akahane, Nature Mater. 4, 207 (2005).

8. L. C. Andreani and D. Gerace, Phys. Rev. B 73, 235114 (2006).

9. W. Bogerts, R. Baets, P. Dumon, V. Wiaux, S. Beckx, D. Taillaert, B. Luyssaert, J. Van Campenhout, P. Bienstman, and D. Van Thourhout, J. Lightwave Technol. 23, 401 (2005).

10. N. Le Thomas, R. Houdré, M. V. Kotlyar, D. O’Brien, and T. F. Krauss, J. Opt. Soc. Am. B 24, 2964 (2007)

11. J. Jágerská, N. Le Thomas, R. Houdré, J. Bolten, C. Moormann, T. Wahlbrink, J. Ctyroký, M. Waldow, and M. Först, Opt. Lett. 32, 2723 (2007).

12. N. Le Thomas, V. Zabelin, R. Houdré, M. V. Kotlyar, and T. F. Krauss, Phys. Rev. B 78, 125301 (2008). 